Methods: In this prospective cohort study, we assessed a total of 84 patients (8 males and 76 females; average age $72.0 \pm 6.0$ years) who underwent a primary unilateral total knee arthroplasty (TKA). Before and 1 month after TKA, patients completed physical performance tests including stair climbing test (SCT), 6 min walk test(6MWT), timed up and go test (TUG), isometric knee flexor and extensor strength of the surgical and non-surgical knees, and instrumental gait analysis for spatiotemporal parameters. Self-reported disease-specific physical function measured by using the Western Ontario McMaster Universities Osteoarthritis Index (WOMAC) and self -reported quality of life measured by using Euro QOL five dimensions (EQ-5D) questionnaire.

Results: In the bivariate analyses, the postoperative SCT-ascent had a significant positive correlation with the SCT-ascent $(r=0.29, p=0.01)$, SCT-descent $(r=0.28, p=0.01)$, TUG $(r=0.37, p<0.01)$, preoperative age $(r=0.25, p=0.02)$, and a significant negative correlation with the preoperative 6MWT $(r=-0.33, p<0.01)$, peak torque (PT) extensor of surgical knee $(r=-0.29, p=0.01)$, PT flexor of surgical knee $(r=-0.26, p=0.02)$, PT extensor of the non-surgical knee $(r=-0.26$, $p=0.02)$, PT flexor of the nonsurgical knee $(r=-0.25, p=0.02)$. The postoperative SCT-descent had a significant positive correlation with the SCT-ascent $(r=0.28$ $p=0.01)$, SCT-descent $(r=0.40, p<0.01)$, TUG $(r=0.38, p<0.01)$, preoperative age $(r=0.27, p=0.01)$, WOMAC function $(r=0.30, p<0.01)$, and a significant negative correlation with 6-MWT $(r=-0.33, p<0.01)$, PT extensor of surgical knee $(r=-0.23$ $p=0.04)$, PT flexor of surgical knee $(r=-0.24, p=0.03)$, PT extensor of the nonsurgical knee $(r=-0.25, p=0.03)$, PT flexor of the nonsurgical knee $(r=-0.23, p=0.03)$. In the linear regression analyses, the postoperative SCT-ascent had a significant positive correlation with the preoperative TUG $(B=0.28, p<0.01)$, PT extensor of surgical knee $(B=-0.23, p=0.03)$ and the postoperative SCT-descent had a significant positive correlation with preoperative SCT-descent $(r=0.28, p=0.01)$, and the age $(B=0.20, p=0.04)$.

Conclusions: This study demonstrated that preoperative physical function influenced on postoperative stair climbing ability 1 month after TKA. Using variables easily measured before surgery, it may be possible to predict with good accuracy for postoperative stair climbing ability. In addition, these results could be of importance in determining variable preoperative rehabilitation strategies to improve stair climbing ability, especially focusing on balance, endurance and strengthening exercises.

Disclosure of Interest: None declared

DOI: 10.1136/annrheumdis-2018-eular.1218

\section{SAT0555 ON THE WAY TO KNEE REPLACEMENT: TRAJECTORY AND CORRELATION OF KNEE OA MRI CARTILAGE THICKNESS, RADIOGRAPHIC JOINT SPACE WIDTH, AND WOMAC KNEE PAIN IN THE OAI}

C.K. Kwoh ${ }^{1}$, E.L. Ashbeck ${ }^{1}$, M.J. Hannon ${ }^{2}$, S. Wax ${ }^{3}$, J. Kraines ${ }^{3} .{ }^{1}$ University of Arizona Arthritis Center, University of Arizona, Arizona; ${ }^{2}$ Medicine, University of Pittsburgh, Pittsburgh; ${ }^{3}$ EMD Serono, Inc, Billerica, USA

Background: Symptoms and structure are both important outcomes in osteoarthritis. However, prior knee osteoarthritis studies have found either poor or no correlation between structural and symptom outcomes.

Objectives: 1) Estimate the trajectory of mean WOMAC pain scores, medial minimum JSW (mJSW), and quantitative total tibiofemoral joint (TFJ) cartilage thickness in the five years leading up to knee replacement $(K R)$, in $K R$ case and control knees; 2) Estimate the correlation between change in WOMAC pain scores and change in structural measures prior to KR.

Methods: Osteoarthritis Initiative participants with at least one knee that met common eligibility criteria for DMOAD RCTs up to the 4 year follow-up visit were selected for analysis. Eligibility criteria included Kellgren and Lawrence grade of 2 or 3 , medial mJSW $\geq 2.5 \mathrm{~mm}$, and knee pain at its worst in the past 30 days (4-9 on a 10-point scale, or 0-3 with pain medication). Quantitative MRI (qMRI) cartilage thickness across the TFJ was measured on 3 Tesla MRI. Medial mJSW was measured on fixed flexion weight-bearing radiographs. WOMAC questionnaires were administered annually, and pain scores were scaled 0-100. Mean pain scores and structural measures at each annual visit were estimated with $95 \%$ confidence intervals $(\mathrm{Cl})$ using mixed effects models. Correlations between changes in structure and WOMAC pain scores were estimated using Spearman correlation coefficients $(r)$, with negative coefficients indicating a correlation between loss of cartilage and increase in pain.

Results: WOMAC scores and medial mJSW measures were available for 91 cases (KR knees) and 1833 controls, with knees contributing an average of 4.5 WOMAC observations and 3.7 medial mJSW observations to the longitudinal analysis. TFJ cartilage thickness measures were available in 86 cases and 524 controls, with knees contributing an average of 3.1 observations. Over 4 years of observation, among knees that went on to $\mathrm{KR}$, the greatest annual change in mean WOMAC pain $(10.8 ; 95 \% \mathrm{Cl}: 7.2,14.4)$ and cartilage thickness $(-0.100 \mathrm{~mm} ; 95 \% \mathrm{Cl}:-0.118,-0.082)$ occurred between the two visits most proximal to $\mathrm{KR}$, while the decrease in mean medial mJSW in the final year $(-0.408$; $95 \% \mathrm{Cl}:-0.545,-0.270$ ) was similar to the rate of loss in prior years (figure 1). Average pain and structure was relatively stable among knees that did not undergo KR. Estimated correlation was moderate between one year change in WOMAC pain and TFJ cartilage thickness among case knees over the year prior to $K R(n=52, r=-0.46)$, with lower correlations at earlier time points based on a more limited sample. Among control knees, the estimated correlation between annual change in WOMAC pain and structure was negligible at all time points $(r<0.1)$.
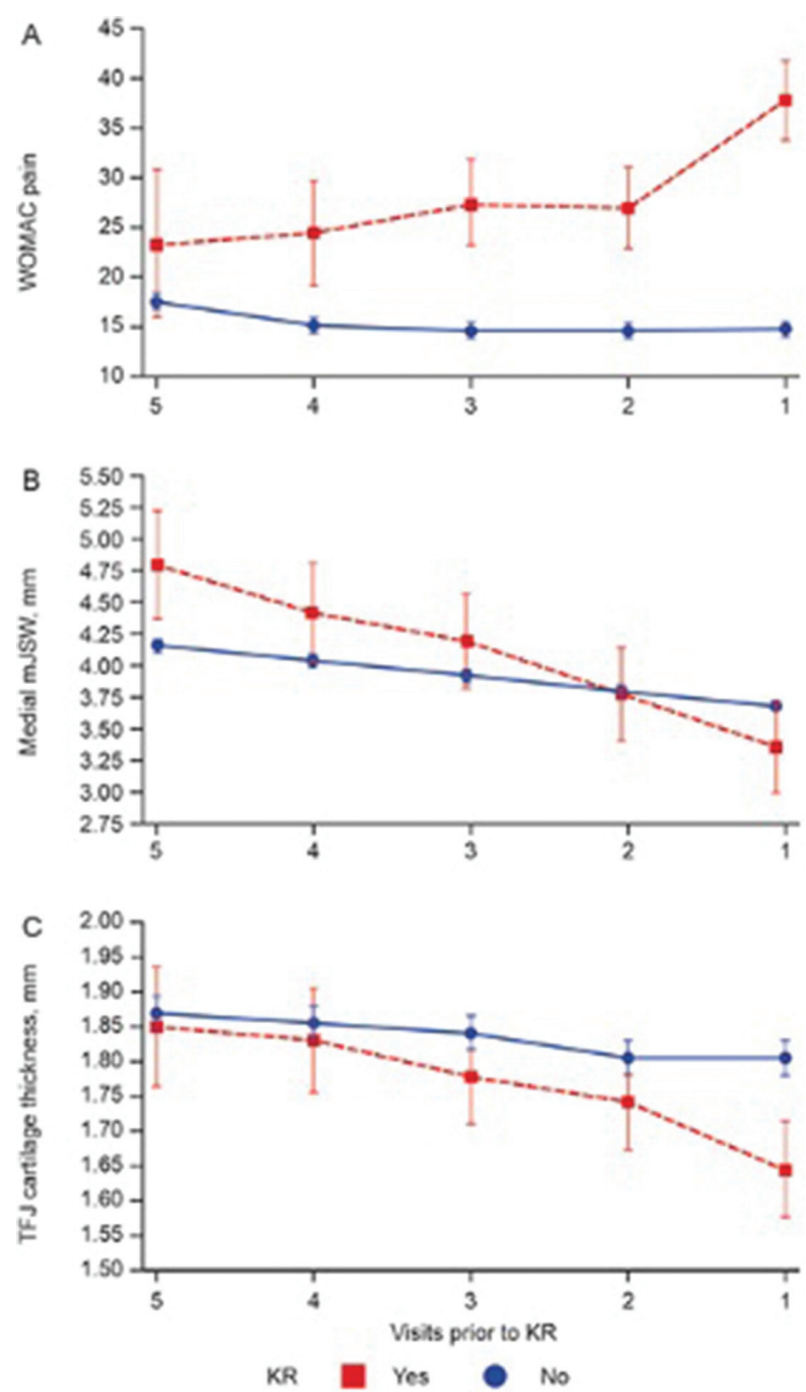

KR, knee replacement: TFJ, total femorosibial joint; WOMAC. Westem Ontario and $/ /$ cMaster Universtios Osteoarthritis Indox

Conclusions: Loss of TFJ cartilage thickness and pain progression were most pronounced over the year prior to knee replacement. Over this time frame, pain progression was moderately correlated with loss of TFJ cartilage, though not with loss of medial mJSW.

Disclosure of Interest: C. K. Kwoh Grant/research support from: Merck KGaA and Abbvie, Consultant for: Astellas, Thusane and Fidia, E. Ashbeck Grant/ research support from: Merck KGaA, M. Hannon Grant/research support from: Merck KGaA, S. Wax Employee of: EMD Serono, Inc, J. Kraines Employee of: EMD Serono, Inc

DOI: 10.1136/annrheumdis-2018-eular.5262 